\title{
Mucosal defect closure using a calibrated, small-caliber-tip, transparent hood after colorectal endoscopic submucosal dissection
}

Complete closure of a mucosal defect using a clip with a transparent cylindrical hood reduces the occurrence of adverse incidents after colorectal endoscopic submucosal dissection (ESD) [1]. Thus, we developed the "reopenable clip over the line method" (ROLM), a technique that uses a line and a reopenable clip (SureClip, 16 mm; MC Medical, Tokyo, Japan) to close a mucosal defect after ESD [2]. With the ROLM, the mucosal defect can be closed completely without clipping its edges on both sides [3]. However, when a cylindrical hood is used, there is a risk that the clip could enter the hood and impede the endoscopic view ( Fig. $\mathbf{1}$ a, b). For this reason, we performed the ROLM using a calibrated, small-caliber-tip, transparent hood (CAST hood; TOP, Tokyo, Japan), to prevent the clips deployed for the mucosal defect closure from interfering with the endoscopic view (ฉ Fig. 1 c, d).

The CAST hood is a tapered hood with a 4-mm tip [4] that allows the clips that are fixed to close the mucosal defect to be deflected out of the hood by the edges of the hood. It can also be used under water to maintain the endoscopic field of view intraoperatively [5].

In our case, the patient had early rectal cancer that was completely resected by ESD. The resected mucosal defect was approximately $5 \mathrm{~cm}$ in size, straddled the folds, and was completely closed using the ROLM with a CAST hood ( Fig. 2; - Video 1). The reopenable clips did not interfere with the endoscopic view during the ROLM. A cylindrical hood with a large diameter is not always necessary because the ROLM allows the mucosal defect to be closed by grasping only the defect and the nearby muscularis propria, without grasping the edges on both sides of the mucosal defect.

Endoscopy_UCTN_Code_TTT_1AO_2AI

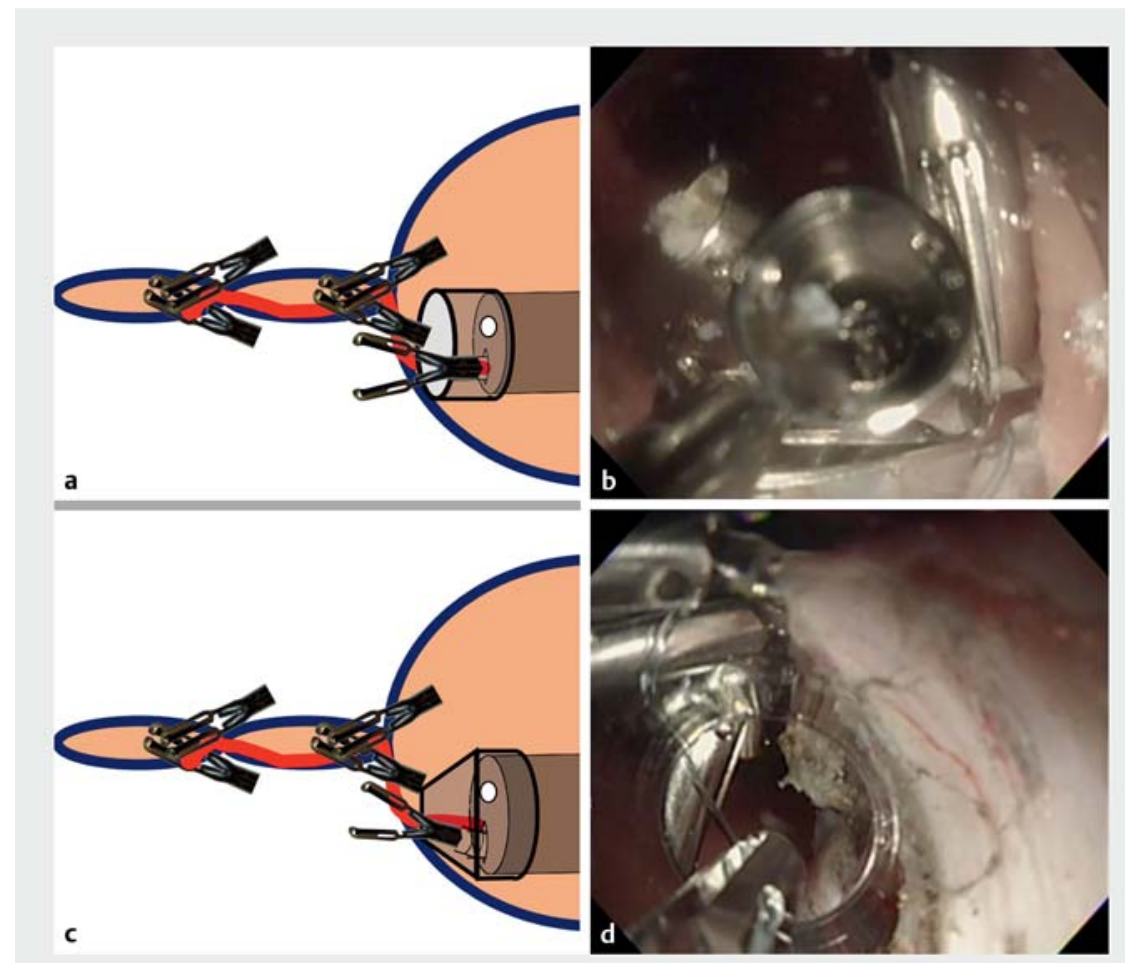

- Fig. 1 Comparison of the endoscopic fields of view using the transparent cylindrical hood and using the calibrated, small-caliber tip, transparent hood (CAST hood) under water. a, b With the cylindrical hood, the clip that is placed just before the endoscope interferes with the endoscopic view. $\mathbf{c}, \mathbf{d}$ With the CAST hood, the clip placed immediately before the endoscope does not obstruct the endoscopic view.

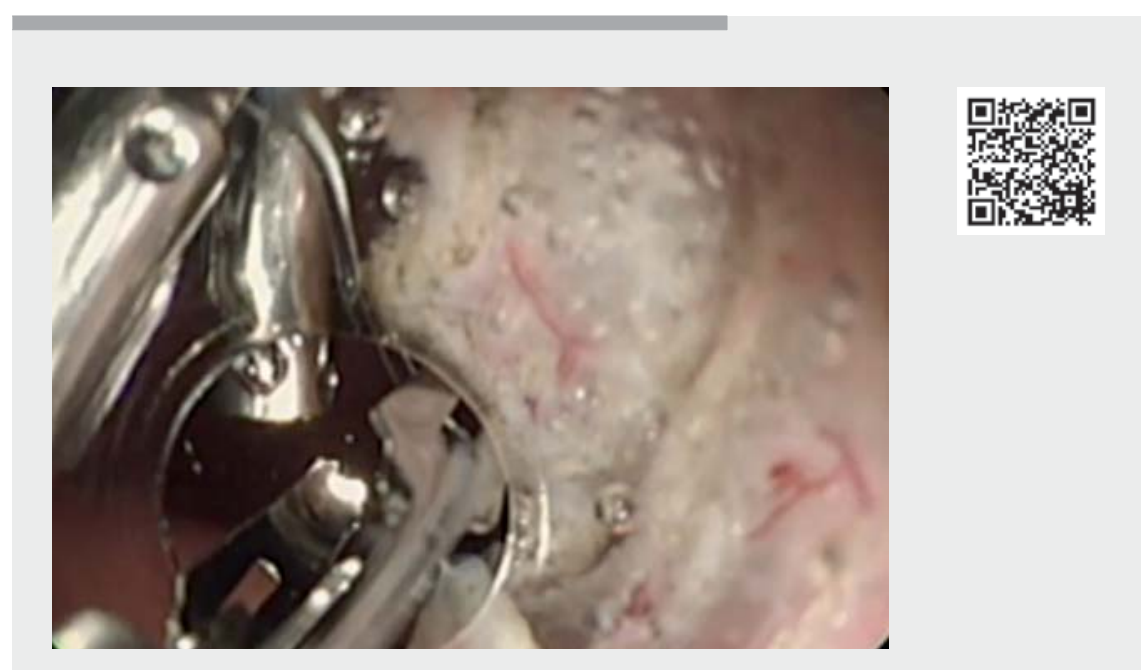

Video 1 Closure of a postendoscopic submucosal dissection mucosal defect using the reopenable clip over the line method and a calibrated, small-caliber-tip, transparent hood. 

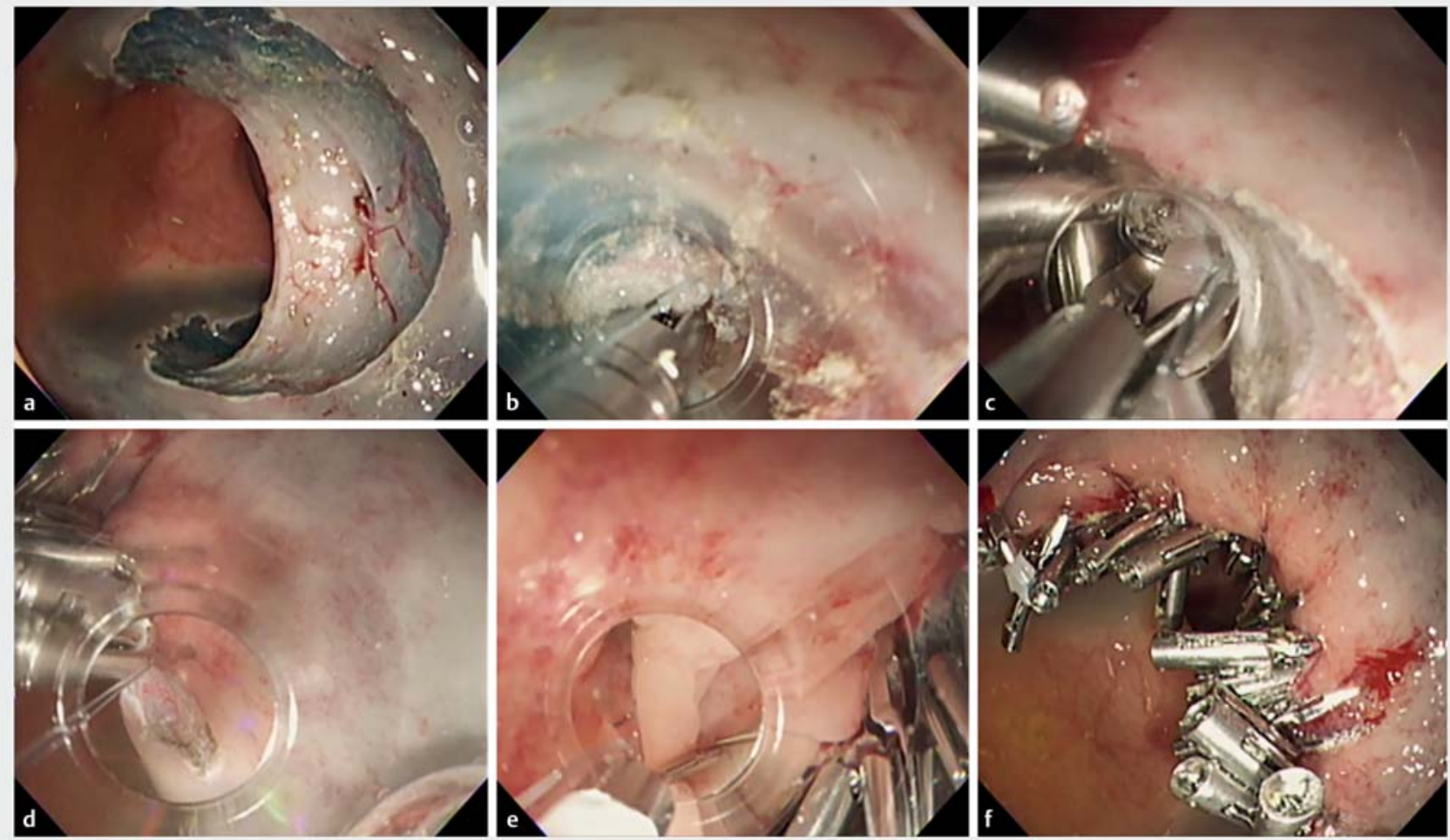

Fig. 2 Example of mucosal defect closure using a calibrated, small-caliber-tip, transparent hood (CAST hood). a The mucosal defect is 50 mm in size and crosses the rectal fold. b First, a clip line is placed on the oral side, grasping the mucosa and muscularis propria. Next, after performing the reopenable clip over the line method (ROLM), a clip is also placed on the anal side on the mucosa and muscularis propria. $\mathbf{c}$ When the CAST hood is used, the clip does not interfere with the endoscopic view under water. $\mathbf{d}$ The mucosal defect is gradually closed by repeated clipping using the ROLM. e The line is fixed to the clip and the line is cut using a modified locking-clip technique. $\mathbf{f}$ The mucosal defect after complete closure using the ROLM.

\section{Competing interests}

The authors declare that they have no conflict of interest.

The authors

Tatsuma Nomura ${ }^{1,2} \odot$, Shinya Sugimoto ${ }^{1}$, Taishi Temma', Jun Oyamada ${ }^{1}$, Keiichi Ito ${ }^{2}$, Akira Kamei ${ }^{1}$

1 Department of Gastroenterology, Ise Red Cross Hospital, Ise, Mie, Japan

2 Department of Gastroenterology, Mie Prefectural Shima Hospital, Shima, Mie, Japan

Corresponding author

\section{Tatsuma Nomura, MD}

Department of Gastroenterology,

Ise Red Cross Hospital, 1-471-2 Funae, Ise, Mie, JP 516-8512, Japan

m06076tn@icloud.com

\section{References}

[1] Albéniz E, Álvarez MA, Espinós JC et al. Clip closure after resection of large colorectal lesions with substantial risk of bleeding. Gastroenterology 2019; 157: 1213-1221

[2] Nomura T, Sugimoto S, Kawabata M et al. Large colorectal mucosal defect closure post-endoscopic submucosal dissection using the reopenable clip over line method and modified locking-clip technique. Endoscopy 2021. doi:10.1055/a-1381-6435

[3] Nomura T, Sugimoto S, Temma T et al. Clipline closure with the reopenable clip over line method for a large mucosal defect after gastric endoscopic submucosal dissection. Endoscopy 2022; 54: E1-E2. doi:10.1055/a1346-8991

[4] Hayashi Y, Yamamoto H, Yano T et al. A calibrated, small-caliber tip, transparent hood to aid endoscopic balloon dilation of intestinal strictures in Crohn's disease: successful use of prototype. Endoscopy 2013; 45: E373-E374
[5] Nomura T, Sugimoto S, Oyamada ] et al. Gl endoscopic submucosal dissection using a calibrated, small-caliber-tip, transparent hood for lesions with fibrosis. VideoGIE 2021; 6: 301-304. doi:10.1016/j. vgie.2021.03.001

\section{Bibliography}

Endoscopy 2022; 54: E691-E692

DOI 10.1055/a-1750-8800

ISSN 0013-726X

published online 18.2.2022

(c) 2022. Thieme. All rights reserved.

Georg Thieme Verlag KG, Rüdigerstraße 14, 70469 Stuttgart, Germany 